\title{
Welches ist der Stellenwert der Luft-Plethysmographie zur Diagnostik der Iliakal-Venen-Obstruktion?
}

\section{What is the value of APG in the diagnosis of iliac vein obstruction?}

Autoren

Erika Mendoza', Christopher R Lattimer ${ }^{2,3}$

Institute

1 Venenpraxis Wunstorf

2 Josef Pflug Vascular Laboratory, Ealing Hospital, Middlesex, UK, Department of Surgery and Cancer, Imperial College, London, UK

3 West London Vascular and Interventional Centre, Northwick Park Hospital, Harrow, UK

Schlüsselwörter

APG, Luft-Plethysmographie, venöse Obstruktion, Stenting der V. iliaca

Key words

APG, air-plethysmography, venous obstruction, iliac vein

stenting, venous drainage index

eingereicht 09.04.2019

akzeptiert 25.04.2019

Bibliografie

DOI https://doi.org/10.1055/a-0895-2425

Online-Publikation: 24.05.2019

Phlebologie 2019; 48: 237-244

(c) Georg Thieme Verlag KG Stuttgart · New York

ISSN 0939-978X

Korrespondenzadresse

Erika Mendoza

Venenpraxis Wunstorf

Speckenstr. 10

31515 Wunstorf

E-Mail: erika.mendoza@t-online.de

Englische Version unter:

https://doi.org/10.1055/a-0895-2425

\section{ZUSAMMENFASSUNG}

Durch die Möglichkeit der Stenteinlage in iliakale Venen scheint die Behandlung der zentralen Obstruktion ein leichtes Unterfangen geworden zu sein. Allerdings bedingt diese Behandlung eine zumindest vorübergehende Antikoagulation - und der Stent wird lebenslang im Bein verbleiben. Und die Evaluation der Ergebnisse ist noch nicht gut etabliert. Auch die Indikationsstellung ist nicht immer objektivierbar gegeben, wenn man bedenkt, dass $25 \%$ der Bevölkerung eine Stenose der linken V. iliaca von über $50 \%$ aufweisen, auch wenn sie meist keine Symptome haben. Erschwerend kommt hinzu, dass die venöse chronische Insuffizienz als klinisches Syndrom (CVI) mehrere Ursachen haben kann, wie Varikose, Obstruktion oder Refluxe der tiefen Achse und die Behandlung eines einzelnen, anatomischen Befundes nicht unbedingt die Klinik nachhaltig verbessern muss. Die Luft-Plethysmographie (Air Plethysmography, APG) bietet in diesem Zusammenhang eine objektive, vom Untersucher und vom Patienten unabhängige Auswertungsmöglichkeit für die venöse Drainage und für die venöse Wiederauffüllzeit, die in jedem Fall vor einem Eingriff der zentralen Venen und zur Evaluation des Ergebnisses gefordert werden sollte.

\section{ABSTRACT}

It looks like treatment of central venous obstruction has become easy since the introduction of iliac stenting procedures. However, this treatment includes a minimum period of anticoagulation and the stent is for life. Furthermore, there are no accepted criteria for evaluation of the results. It is difficult also to establish robust indications of stenting especially since $25 \%$ of the normal population have a diameter reduction $>50 \%$ in the left iliac vein.

Another confounding fact is that chronic venous insufficiency (CVI) can be the consequence of multiple simultaneous pathologies, like varicose veins, obstruction and deep venous reflux. Thus, the treatment of one of these may not have a significant clinical effect if the cause is multifactorial.

Air Plethysmography (APG) is an objective, non-invasive and patient independent investigation of venous drainage. This includes gravitational filling (venous filling index) and elevation emptying (venous drainage index) all in the same test. This investigation should be performed as a screening test, prior to deep venous stenting and also as a follow-up instrument. 


\section{Einleitung}

Als Behandlung einer angeborenen Obstruktion der Iliakalvenen, wie dem May-Turner Syndrom, oder der postthrombotischen Okklusion erfährt das Einlegen eines Stents einen großen Zuspruch bei Phlebologen, Gefäßchirurgen, Angiologen und interventionellen Radiologen. Es wird als leicht durchzuführender Eingriff mit wenig Nebenwirkungen dargestellt und verspricht schnelle Linderung von Stauungsbeschwerden. Es wurde sogar als Therapieoption für Patienten mit großlumigen Krampfadern angeboten [1] und mit dieser Option sogar in Werbebroschüren von einigen Praxen, auch in Deutschland, dem Patienten angeboten. Allerdings konnte kein Zusammenhang zwischen großlumigen Varizen und einer Iliakalvenen-Obstruktion bei Patienten ohne Thrombose-Vorgeschichte nachgewiesen werden [2]. Bedenkt man, wie intensiv auf der ganzen Welt über die Stenting-Verfahren diskutiert wird (nicht nur in Publikationen und auf Kongressen, sondern auch in internationalen ärztlichen Whats-App Gruppen und Mail Austauschgruppen), überrascht es, dass wir keine standardisierten Verfahren haben, um die Qualität der Indikation zum Einsetzen eines Stents sowie das Ergebnis nach erfolgtem Eingriff zu prüfen. Abgesehen von dem technischen Sofortergebnis der „Offenheit“ des Gefäßes nach Stenteinlage, gibt es sonst wenige Kriterien um das Ergebnis auszuwerten. Immerhin handelt es sich um das Einbringen eines Fermdkörpers, der lebenslang bleibt. Dies hat außerdem zur Folge, dass man vorübergehend oder dauerhaft Medikamente zur Gerinnungshemmung einnehmen muss. Daher wäre es wichtig, diese rasante Entwicklung in der Phlebologie neu zu überdenken.

\section{Allgemeine Überlegungen zur Obstruktion tiefer Beinvenen}

Ein Verschluss oder die Verengung der tiefen Beinvenen, besonders deren proximaler Segmente, wie die V. femoralis communis oder die V. iliaca, sei es durch Thrombose, Stenose, Fehlbildung oder Kompression von außen, behindert den venösen Abstrom aus dem Bein. Das kann je nach Ausprägung des Abfluss-Hindernisses eine schwere venöse Insuffizienz und eine Verschlechterung der Lebensqualität zur Folge haben. Die Schwierigkeit besteht darin, zu entscheiden, ob die Lumenreduktion der Grund für die venösen Beschwerden ist, da eine Einengung von mehr als $50 \%$ des Lumens der linken V. iliaca bei $25 \%$ der durchaus auch asymptomatischen Bevölkerung zu finden ist [3]. Diese Häufigkeit erlaubt auch die Annahme, dass es sich bei der mit diagnostischen Mitteln darstellbaren Einengung um eine physiologische Situation ohne zwingende klinische Konsequenz handelt.

Die Evaluation der iliakalen Stenose mittels Duplex erfordert gute Kenntnisse und kann durch anatomische Gegebenheiten wie Übergewicht oder zusätzliche Kompression durch den Schallkopf selbst beeinflusst sein.

Der intravasale Ultraschall (IVUS) als primäres Diagnostikum einzusetzen ist nicht optimal, da es sich um ein invasives, teures Verfahren handelt, das nicht als Screening Methode geeignet ist.

Sämtliche bildgebenden Verfahren können ohnehin die hämodynamischen Konsequenzen der Läsion nicht adäquat auswerten, da sie entweder nicht die Kollateralen erfasst (IVUS) oder dies nur unter technisch hohem Aufwand, wenn überhaupt erreichbar ist
(Ultraschall). Die Schnittbild-Verfahren (MRT, CT) stellen Kollateralvenen, besonders wenn sie sich korkenzieherartig anordnen auch nicht immer nachvollziehbar dar und bei Phlebographien ist die Kontrastmittelanfärbung in Beckenvenen limitiert [4].

Außerdem ist es sehr selten, einen Patienten mit isolierter Beckenvenenläsion zu finden, es sei denn, diese verursacht keine Klinik - allerdings müsste man sie ja in diesem Fall auch nicht behandeln... Bei symptomatischen Läsionen der Beckenvenen finden wir in der Regel auch Refluxe - in den oberflächlichen Venen bei Varikose oder den tiefen Venen bei Postthrombotischen Syndrom sowie oft auch eine Reduktion des Venenwand-Tonus in den ReservoirVenen der Waden nach Thrombose und eingeschränkter Muskelpumpfunktion. Und dann stellt sich die Frage, ob die Korrektur der nicht-obstruktiven Komponenten des komplexen Erkrankungsbildes nicht weniger invasiv wäre als die Behandlung der Obstruktion [4]. Dies bedeutet die Verbesserung der Muskelpumpe mit Kompressionsstrümpfen [5] sowie Trainings-Programmen und Physiotherapie für die Aktivierung der Muskelpumpe. Die Behandlung der Varikose - allerdings erst nach Sicherstellung, dass die Stammvenen nicht der Drainage der tiefen Beinvenen als Bypass dienen. Dies kann man durch Analyse der Flusskurve oder mit der Net-Flow-Messung im Duplex durchführen [6]. Ein weiteres Verfahren, um den Gewinn einer Behandlung der V. saphena magna vorherzusagen, ist die Luft-Plethysmographie (Air Plethysmography, APG) unter Kompression der Stammvene (s.u.).

\section{Allgemeine Informationen zur Luft Plethysmographie (APG)}

Die Luft-Plethysmographie kann durch Assistenzpersonal durchgeführt werden. Sie ist nicht invasiv und nicht schmerzhaft. Sie misst Volumenveränderungen der Wade. Dazu legt man um die Wade eine Luftmanschette, die an einen Druckabnehmer gekoppelt ist. Somit werden die absoluten Volumenänderungen in $\mathrm{ml}$ registriert und auf einer Zeitachse dargestellt, sodass die Änderungsrate des Volumens in $\mathrm{ml} / \mathrm{s}$ errechnet werden kann. Um die Drainage der Venen des Beins zu testen, wird das Bein schnell angehoben. Dadurch entleert sich das Blut über die Venen des Beins und die Beckenvenen zur V. cava hin. Die Wade verringert in der Folge ihr Volumen. Um die Klappenkompetenz zu testen wird der Patient aufgesetzt oder hingestellt mit hängendem Bein. Dabei wird die Zeit bis zur Wiederauffüllung des ursprünglichen Volumens gemessen. Die absolute Änderung in ml, sowie die Rate der Änderung mit ml/s wird errechnet. Die Drainagegeschwindigkeit korreliert umgekehrt mit dem Grad der Obstruktion und die Wiederauffüllgeschwindigkeit mit dem Grad der Klappen-Inkompetenz. Eine schnelle Entleerung bei nicht-obstruktiv veränderten Beinen verursacht eine Kurve mit der Form eines Wasserfalls [2]. Bereits Trendelenburg hat dies beschrieben. Die erste Veröffentlichung mit Luft-Plethysmographie-Änderungen des Beinvolumens auf einem Kipptisch stammt von J.C. Allan, aus dem Jahr 1964 [7].

Schließlich erlaubt die Plethysmographie das Errechnen des so genannten „Working Volume“ - des Volumens, um das das Bein sich entleert und füllt, jedoch nicht des gesamten venösen Volumens.

Die Lageänderung von Liegend mit erhobenem Bein zu Sitzend mit hängendem Bein oder Stehend kann in einem normalen Unter- 

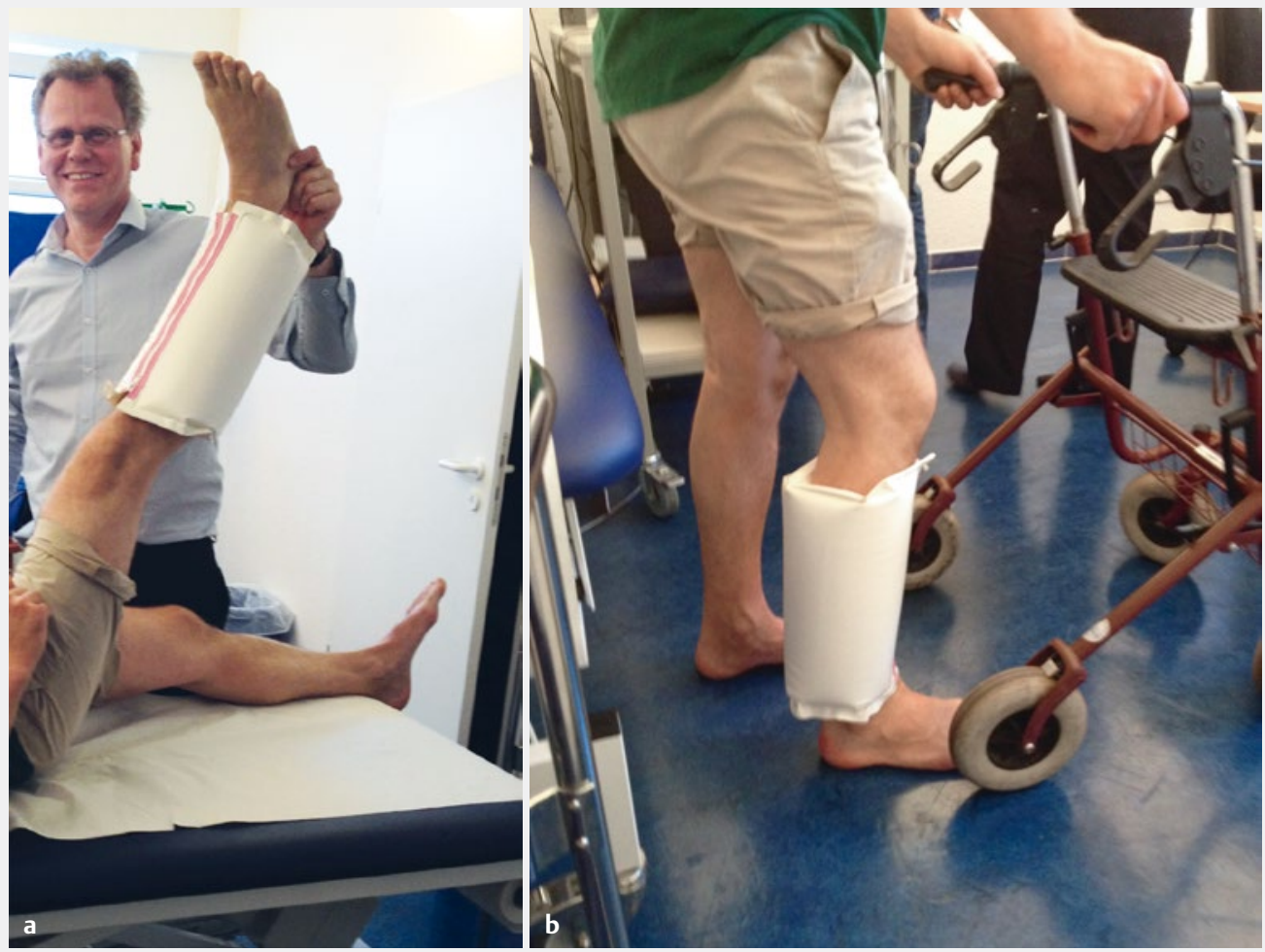

- Abb. 1 Durchführung der Luft-Plethysmographie im Erkundungsraum. a Liegender Patient mit der Manschette um die Wade mit angehobenem Bein, vom Untersucher (CL) getragen. b Patient im Stehen mit festen Händen an den Händen, um Bewegungen zu vermeiden.

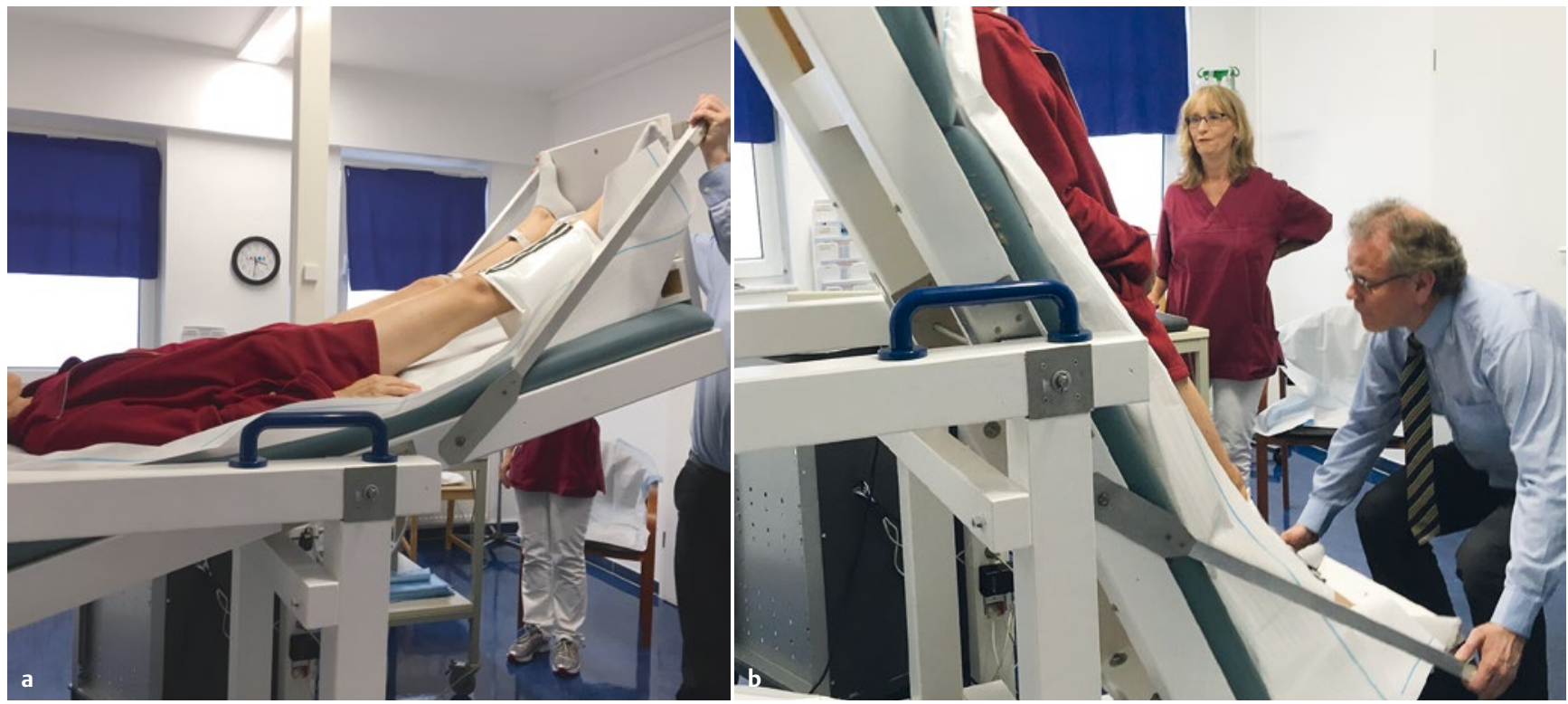

> Abb. 2 Luft-Plethysmographie auf einem handbetätigten Kipptisch durchführen. a Trendelenburg bei $-45^{\circ}$. b Stehende Position.

suchungsraum erfolgen ( $\vee$ Abb. $\mathbf{1}$ ) oder unter Zuhilfenahme eines händisch betriebenen Kipptisches ( $\mathbf{A b b}$. 2). Kipptische mit Motorantrieb sind nicht sinnvoll, da diese für die Lageänderung mehr als 15 Sekunden benötigen. Das ist viel zu lang - die Entleerung eines gesunden Beins geschieht in wenigen Sekunden.
Auf einem Bildschirm wird die Kurve der Beinfüllung registriert (s. - Abb. 3). Bei kompletter Entleerung und kompletter Füllung wird ein Plateau erreicht. Als Kardinalpunkte werden auf der Kurve die Plateau-Linie bei Füllung und bei Entleerung gewählt, sowie der Moment, in dem das Bein gehoben wird und der Moment, an 


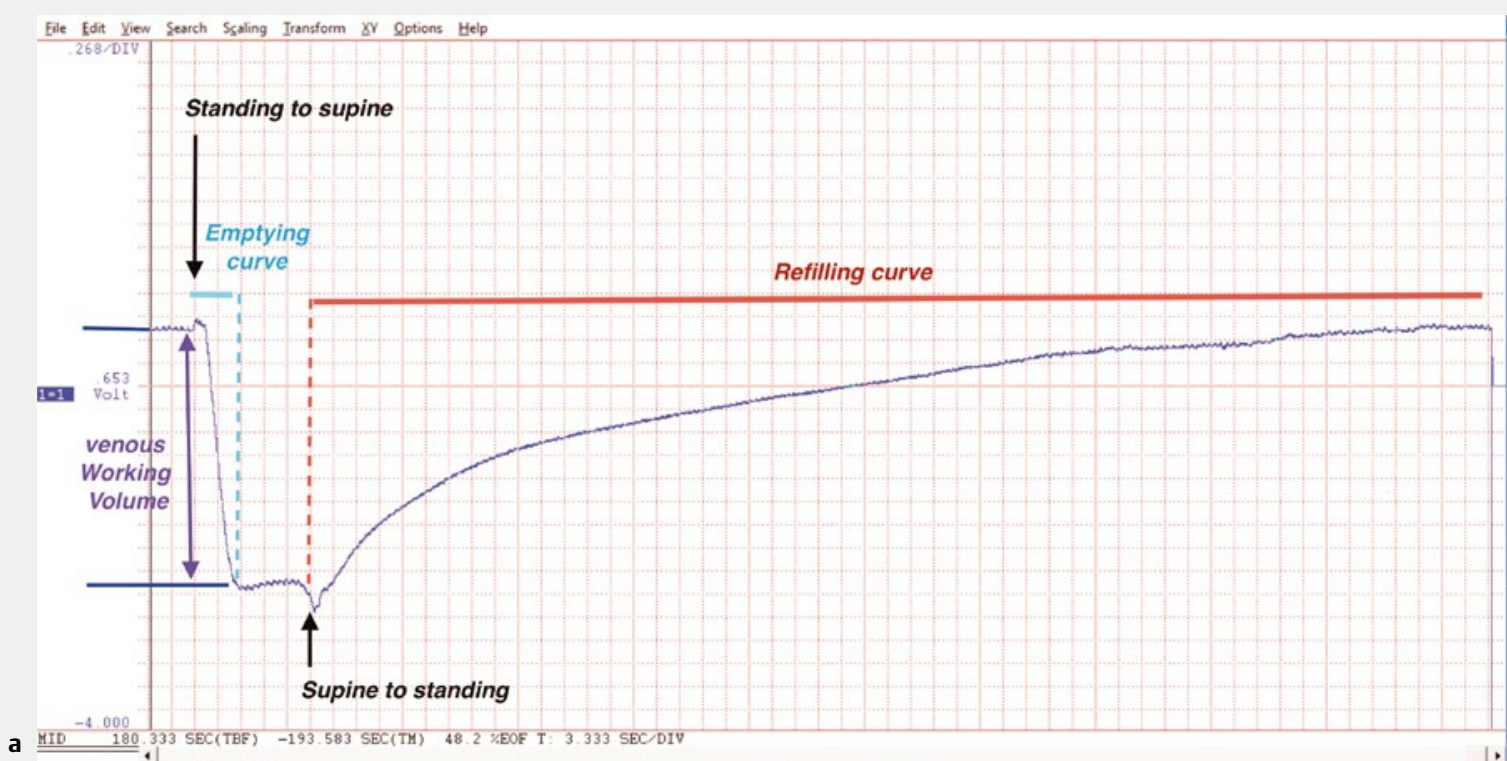

a
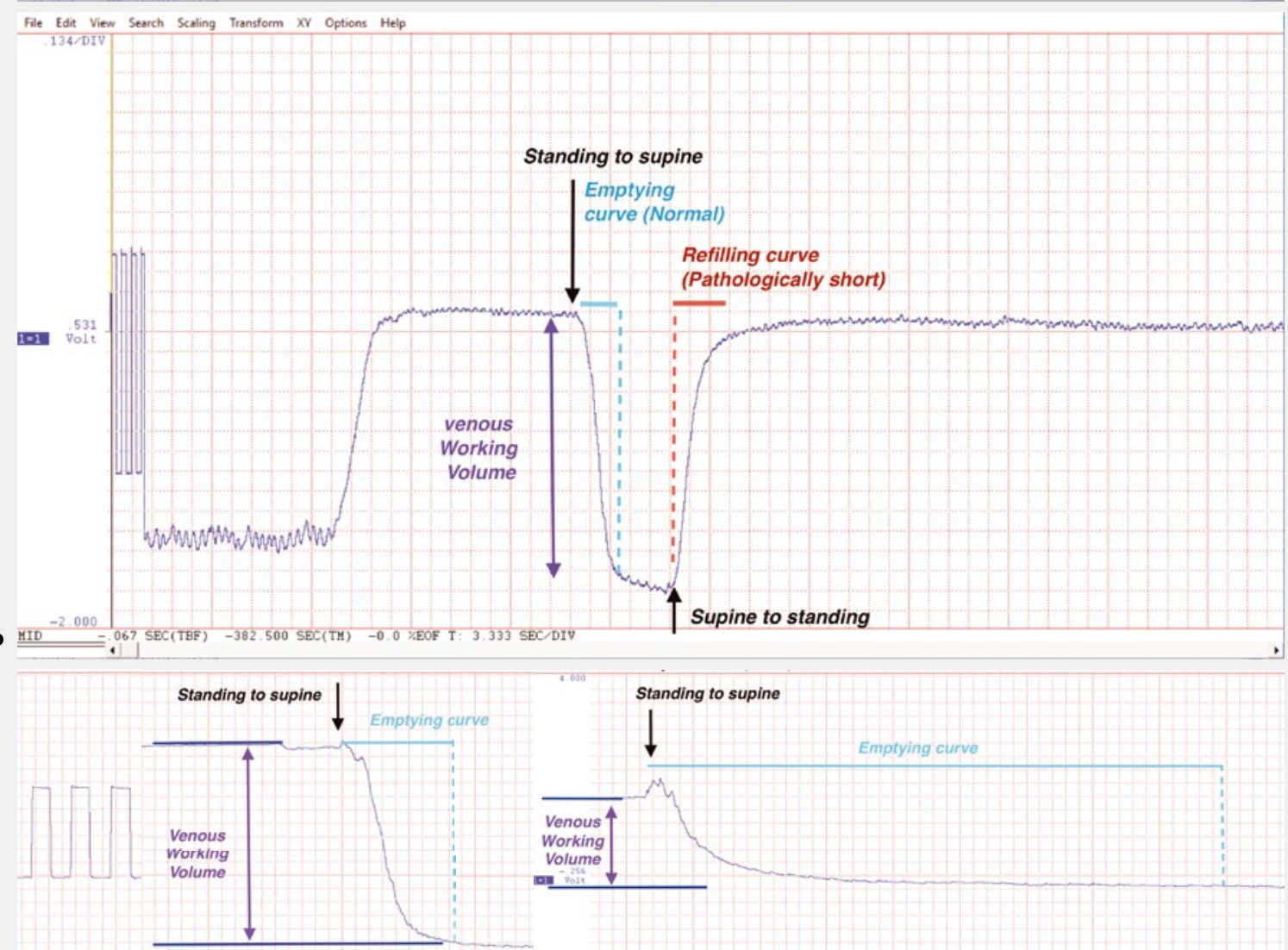

Cali-

bration

Native

Obstruction with $40 \mathrm{~mm} \mathrm{Hg}$

c

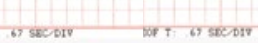

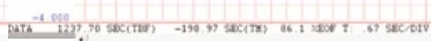

-Abb.3 APG-Kurven mit Entleerung des Beines nach dem Bewegen des Patienten vom Stehen in die Trendelenburg und Nachfüllen der Kurve nach dem Bewegen des Patienten vom Trendelenburg zum Stehen. a Normalkurve mit schneller Entleerung (kein Hindernis) und langsamer Wiederbefüllung (keine Ventilinkompetenz). b Pathologische Kurve mit schneller Entleerung (keine Behinderung) und schnellem Nachfüllen (große Klappeninkompetenz). c Pathologische Kurve mit langsamer Entleerung (Verstopfung), die sich bei stehendem Bein von $70^{\circ}$ in Rückenlage bewegt. * mit erhöhten $70^{\circ}$ Beinen.

Standing to supine = Von stehend zu Kopftieflage, Supine to standing: Von Kopftieflage zu Stehend, Emtying curve: Drainage-Curve, RefillingCurve $=$ Wiederauffüllungs-Kurve, Venous Working Volume $=$ Venöses Arbeitsvolumen. Native $=$ ohne Manschette, Obstruction $=$ Druckmanschette auf $40 \mathrm{~mm} \mathrm{Hg}$. 


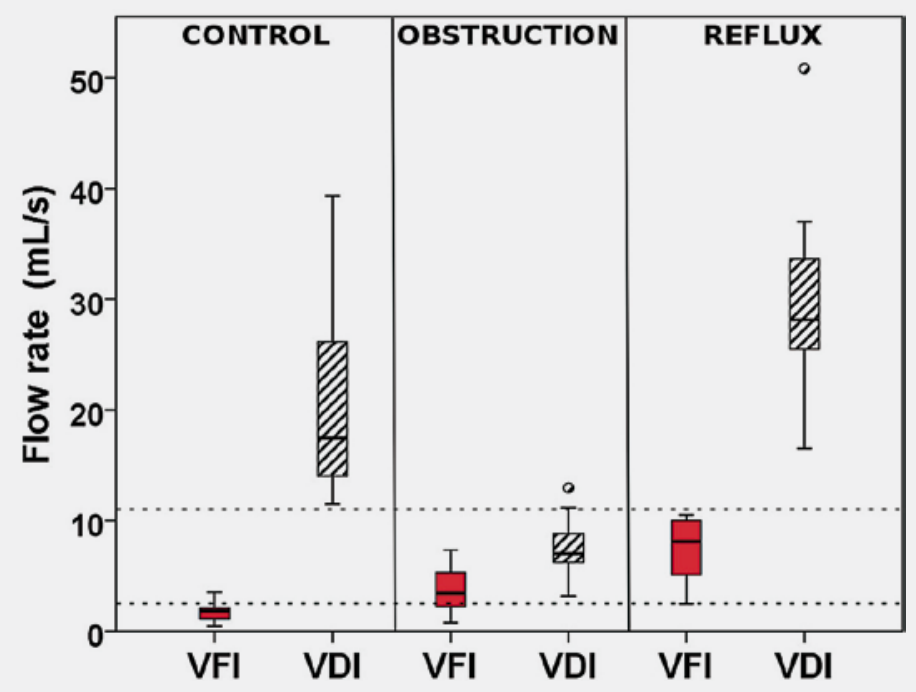

- Abb. 4 Box-Diagramme zum Vergleich der Ergebnisse der APG auf dem Tilt Table. Venenfüllindex (VFI) und Venenabflussindex (VDI) zwischen drei Probandengruppen: Gesunde Kontrollen, Patienten mit Obstruktion der Iliakalvene und Patienten mit großen saphenösen Venen des Refluxins. Die horizontalen gestrichelten Linien repräsentieren 10,8 ml/s (untere Grenze) und 2,9 ml/s (obere Grenze). Dies sind empfohlene Grenzwerte für Obstruktion bzw. Rückfluss. (Daten aus [8])

- Tab. 1 Ergebnisse der Kipptabelle mit Luft-Plethysmographie in den 3 Studiengruppen ( $n=11 /$ Gruppe). Ausgedrückt als Median (Interquartilbereich) [8].

\begin{tabular}{|c|c|c|c|c|c|}
\hline & Kontrolle & Obstruktion & $\begin{array}{l}\mathrm{p}^{\mathrm{a}} \\
\text { (Kontrolle vs. } \\
\text { Behinderung) }\end{array}$ & Reflux & $\begin{array}{l}\text { p value a } \\
\text { (Kontrolle } \\
\text { vs. Reflux) }\end{array}$ \\
\hline Arbeitsvenösvolumen, wVV (mL) & $138(119-198)$ & $117(80-154)$ & $<, 0005$ & $202(180-240)$ &, 008 \\
\hline Venöse Drainagezeit, VDT90 $(\mathrm{s})$ & $4,9(4,3-5,9)$ & $9,7(8,5-12,9)$ & $<, 0005$ & $5,2(4,4-5,9)$ & ,622 \\
\hline Venöse Füllzeit, VFT90'b (s) & $69,4(33,5-135,3)$ & $33,7(14,4-39,5)$ & 014 & $21(16,6-36,3)$ &, 004 \\
\hline Venöser Drainageindex, VDI (mL/s) & $17,4(13,9-27,2)$ & $7(6-9)$ & $<, 0005$ & $28,1(25,4-34,4)$ & $<, 0005$ \\
\hline Venöser Füllungsindex, VFI (mL/s) & $1,8(1-2,1)$ & $3,4(2,1-5,4)$ &, 014 & $8,1(4,2-10)$ & $<, 0005$ \\
\hline
\end{tabular}

dem das Bein wieder gesenkt wird, daraus errechnet das System das Arbeitsvolumen (working Venous Volume wVV), den venösen Füllungsindex (Venous Filling Index VFI) und den venösen Drainage Index (Venous Draining Index VDI).

Da die Kurven in einer langen Plateau-Phase enden, ist es schwer, den exakten Moment der kompletten Füllung zu definieren. Daher wird das Volumen festgelegt, bei dem das Plateau erreicht wurde und davon $90 \%$ berechnet. Auf der Kurve wird die Zeit abgelesen, zu der $90 \%$ des Volumens erreicht wurde. Und somit wird der Index in $\mathrm{mL} / \mathrm{s}$ errechnet aus der Zeit, die nötig ist, um $90 \%$ des venösen Arbeitsvolumens zu füllen oder zu leeren.

Eine eindeutig pathologische Drainage liegt bei einem venösen Drainage Index (VDI) von unter $10,8 \mathrm{ml} / \mathrm{s}$ vor, eindeutig pathologische Füllungswerte bei einem venösen Füllungsindex (VFI) von über $2,9 \mathrm{ml} / \mathrm{s}$ ( $\triangleright$ Tab. 1 und $\triangleright$ Abb. 4) [8].
Die Messung ist unabhängig von den Hautveränderungen, der Raumtemperatur, der Muskelpump-Fähigkeit des Patienten, seine Fähigkeit, zu kooperieren - was alles die PPG Werte beeinflussen kann [9]. Einschränkungen gibt es allein bei Patienten mit Schwindelgefühl oder mit Schwierigkeiten bei der Beweglichkeit, wenn kein Kipptisch verfügbar ist.

\section{Validierungsstudien der Luft- Plethysmographie (APG)}

Es gab 3 Validierungsstudien für die APG. In der ersten Studie wurde das Luft-Plethysmographie-Gerät an der Wade angeschlossen und am Oberschenkel eine Manschette, wie man sie von der VerschlussPlethysmographie her kennt. An gesunden Probanden wurde eine standardisierte Okklusion am Oberschenkel verursacht und darun- 
ter mittels APG die Drainage gemessen (VDI). Der Drainage-Index wird signifikant gesenkt je höher der Druck der proximalen Manschette steigt, was die Sensitivität des VDI auf proximale Obstruktion bestätigt ( $\triangleright$ Abb. $\mathbf{3 c}$ rechts) [10].

In einer zweiten Studie wurde auf einem manuell betätigtem Kipptisch ( $\mathbf{A}$ bb. 2) der Drainage Index bei gesunden Probanden, Patienten mit ausgeprägter Varikose und ohne Vorgeschichte einer Thrombose und Patienten mit bekannter Obstruktion der Iliakalvenen verglichen [8]. Der VDI war signifikant niedriger bei den Patienten mit Obstruktion als bei den Kontrollprobanden oder auch bei den Patienten mit Varikose mit einem Cut-off Wert von $10,8 \mathrm{~mL} / \mathrm{s}$ ( Abb. 4).

Die dritte Studie untersuchte die Änderungen des VDI in einer kleinen Gruppe mit nicht-thrombotischen Iliakal-Venen Läsionen (NIVL) vor und nach einem Stenting. Der Wert verbesserte sich durch den Eingriff signifikant [11].

\section{Diagnostik der Obstruktion}

Die Symptome der CVI sind sehr unspezifisch: Schweregefühl, Schmerz, Schwellung, Juckreiz und Krämpfe. Sie treten nicht nur bei Patienten mit Verschluss der tiefen Beinvenen auf, sondern auch bei Reflux in den oberflächlichen Beinvenen, sowie auch in der gesunden Bevölkerung [12, 13]: Unabhängig von der Existenz einer venösen Insuffizienz werden die meisten Menschen nach längerem Stehen Symptome entwickeln, die mit einer chronischen venösen Insuffizienz kompatibel sind und sie werden von Kompression profitieren, um diesen Symptomen zu begegnen [13]. Die Ursache der Symptome könnte daher nicht-venös sein, auch wenn eine Obstruktion oder venöse Insuffizienz vorliegt. Darüber hinaus gibt es keine gute Korrelation zwischen „venösen“ Symptomen und klinischen Zeichen der CVI. Ungefähr 20 \% der Patienten mit einem Ulcus cruris venosum haben keine sichtbaren Varizen [14] und weitere $20 \%$ haben keine Schmerzen [15].

Zur Diagnostik und Evaluation bei PTS wurde der Villalta Score eingeführt und auch oft verwendet. Als Instrument zum Messen der Klinik ist der Villalta Score jedoch nicht zuverlässig [16]. Er ist nicht spezifisch, da $42 \%$ der Patienten mit nicht thrombotischen venösen Erkrankungen im Villalta Score Werte über dem Grenzwert für PTS zeigen, auch wenn sie niemals eine Thrombose hatten noch ein Reflux in der tiefen Beinvene nachweisbar ist [17]. Außerdem werden einige spezifische Symptome, wie die venöse Claudicatio bei Obstruktion, nicht abgefragt, ebenso wenig einige diagnostische Zeichen wie Kollateralen auf der Bauchdecke [18].

\section{Warum sollte die APG eingesetzt werden als Screening-Methode für venöse Erkrankungen?}

Die Haupt-Aufgabe der Venen ist die Drainage des Bluts. Und diese Aufgabe ist beeinträchtigt durch Obstruktion und Klappeninsuffizienz. APG misst beide sehr genau, auf eine sehr einfache Art und Weise und ohne Artefakte. Die APG ist verlässlich, unabhängig vom Stadium der Behandlung (prä- oder postoperativ), unabhängig vom Untersucher und wiederholbar. Es handelt sich also um einen objektiven Test der das Ausmaß der hämodynamischen Läsion - sei es Obstruktion oder Klappeninsuffizienz - quantifiziert und der als Follow-up Verfahren eingesetzt werden kann.

In vielen Ländern gibt es ein Gesundheitswesen, das sich unter anderem auf Kliniken und Praxen mit Selbstverwaltung begründet. Diese Kliniken tragen teilweise oder ganz ihr finanzielles Risiko wie jedes Unternehmen. In diesem Setting kann es geschehen, dass Behandlungsentscheidungen nicht nur wegen des Wohlergehens von Patienten getroffen werden. Daher ist es umso wichtiger über einfache und objektive Messmethoden zu verfügen, die zur Indikationssicherung vor invasiven Behandlungsoptionen eingesetzt werden können.

In Deutschland wurde zur Bestimmung eines akuten oder chronischen proximal gelegenen venösen Verschlusses der venöse Abstrom mittels Venenverschlussplethysmographie (VVP) im Liegen gemessen. Sie bestimmt die Änderungen der Kapillarfüllung an der Wade mittels Photo-Plethysmographie oder die Volumenänderungen über Strain-Gauge Systeme zunächst unter zunehmendem Verschluss der Gefäße am Oberschenkeln mit einer pneumatisch bis auf $80 \mathrm{mmHg}$ gefüllten Manschette, die dann plötzlich entleert wurde (Entleerungszeit der Manschette unter 1 Sekunde). Die Geschwindigkeit, in der sich das Volumen der Wade entleert wird indirekt über den Umfang oder die Kapillarfüllung gemessen und wird in Volumen \% angegeben. Leider wurde gezeigt, dass hiermit proximale Abflusshindernisse nicht adäquat nachgewiesen werden konnten [19]. Neue Untersuchungen mittels APG und proximaler pneumatischer Oberschenkelobstruktion haben bewiesen, dass das Tragen von Oberschenkelstrümpfen den venösen Abfluss von $44 \%$ auf $63 \%$ steigert, trotz gleichbleibender zentraler Obstruktion [5].

Auf der anderen Seite wurde bewiesen, dass der Venöse Drainageindex mittels APG eine signifikante Änderung durch Stenting der zentralen Obstruktion erfährt [11] sowie auch bei experimenteller externer proximalen Obstruktion (Druckmanschette) [10].

Die Manöver, die bei der APG angewendet werden, untersuchen die Auswirkung der Schwerkraft auf Lageänderung des Beins. Die Messung ist unabhängig vom Untersucher, sie hängt nicht z. B. von der Handgröße oder der Kraft der Hand wie bei der manuellen Kompression der Wade im Ultraschall ab und auch von der Erfahrung des Untersuchers. Anders als einzelne Kriterien, wie der Durchmesser der Stammvenen, der nach der Operation nicht mehr darstellbar ist, wenn die Vene entfernt wurde, kann durch APG trotzdem auch nach jeder Form von Behandlung objektiviert werden, wie ausgeprägt die venöse Insuffizienz oder Obstruktion im Bein ist. Außerdem ist die Messung unabhängig von der Muskelkraft des Patienten oder seiner Fähigkeit bei Bewegungen oder Atmungen mit zu machen.

Selbstverständlich muss nach der Feststellung einer Abflussbehinderung oder einer venösen Insuffizienz mittels APG als Screening Verfahren ein bildgebendes Verfahren eingesetzt werden, um Anatomie, Lokalisation und Hämodynamik weiter zu definieren, auch weil je nach Erscheinungsform unterschiedliche Behandlungsstrategien zur Verfügung stehen - die dann wiederum anschießend mittels APG evaluiert werden können.

\section{APG gegen unangemessenes Stenting}

Wenn der Patient heutzutage klinische Zeichen und Symptome zeigt, die zu einer Obstruktion passen (definiert als über $50 \%$-ige Einengung des Durchmessers, was immerhin bei $25 \%$ der Bevölke- 
rung auch ohne Symptome zu finden ist [20]) ist die Chance groß, dass er einen venösen Stent erhält als eine moderne Therapieoption.

Abhängig vom Hintergrund des Zentrums oder der Spezialisierung des Therapeuten kann dies zu einer Überindikation zum Stenting führen, da beides, Symptome und Obstruktion in der allgemeinen Bevölkerung recht häufig ist. Aber: Einen Stent setzt man für ein Leben. Und: Er bedingt zumindest vorübergehend orale Antikoagulation oder Antiaggregation.

Waren die Symptome jedoch nicht durch die Obstruktion bedingt, wird der Patient keine Verbesserung spüren. Das Ziel, APG vor einem Eingriff einzusetzen, ist es, zu bestimmen, ob die Lumen-Einengung hämodynamisch signifikant ist. Finden wir keine Veränderung des Abflusses im APG, besteht keine Indikation zur Platzierung eines Stents.

Nach dem Einsetzen des Stents muss der venöse Drainageindex (VDI) sich normalisieren oder verbessern - was natürlich voraussetzt, dass er davor pathologisch war. Üblicherweise ist es aber so, dass der Arzt, der den Eingriff durchführt, nur den technischen Erfolg mit einer Öffnung der Vene im Röntgen wahrnimmt, aber nicht die Klinik nachverfolgt. Leider zeigen die Studien, dass der reine technische Erfolg nicht unbedingt mit einer Verbesserung der Klinik korreliert [21]. Eine Verbesserung objektivierbarer Messgrößen, wie des VDI, würde hier helfen.

Außerdem finden wir meist Patienten mit kombiniertem obstruktiven und refluxiven Anteilen in ihrer Pathologie. In diesen Fällen muss zunächst überdacht werden, welche Krankheit zuerst behandelt werden sollte. Das Einsetzen eines Stents einer hämodynamisch relevanten Läsion kann die Symptome verringern, zumal möglicherweise das venöse Volumen des Beins verringert wird - und damit auch das Volumen im oberflächlichen Venensystem. Auf der anderen Seite könnte auch zunächst der oberflächliche venöse Reflux behandelt werden, da dieser Eingriff weniger invasiv ist. Wenn dadurch die Verbesserung der Klinik ausreichend ist, wäre eine Behandlung der iliakalen Läsion nicht nötig - und möglicherweise war die Auswirkung der Obstruktion dann auch nicht so ausgeprägt wie ursprünglich angenommen.

Außerdem gibt es Situationen mit einem Postthrombotischen Syndrom (PTS) in Kombination mit einem Reflux in der V. saphena magna (VSM). Im Duplex-Ultraschall kann herausgefunden werden, ob die VSM als Drainage für die tiefen Beinvenen dient und somit einen Bypass zum tiefen Venensystem darstellt oder ob hier ein reiner Reflux vorliegt. Im ersten Fall wird die VSM nicht behandelt werden. Eine weitere Möglichkeit dies herauszufinden ist die Untersuchung des so genannten „net flow“ im Duplex [6] oder die Durchführung einer APG (VDI und VFI) ohne und mit Okklusion der VSM unter Duplex-Kontrolle, um den Einfluss des Verschlusses der VSM auf die Drainage und die venöse Füllung zu messen.

Es gab die Idee, dass die iliakale Obstruktion die Ursache für besonders ausgeprägte Krampfadern sein könnte, so zusagen als eine Dilatation in Folge einer proximalen Obstruktion [1]. Allerdings vergisst dies Modell, dass der Volumenüberschuss bei der Varikose dem Reflux und nicht der Obstruktion geschuldet ist. Im APG konnte darüber hinaus das Gegenteil bewiesen werden ( $\mathbf{A} \mathbf{b b} \mathbf{4}$ und $>$ Tab. 1): Die Drainage nach Anheben des Beins war bei ausgeprägter Varikose sogar noch höher als bei Kontrollpatienten (und sowieso als bei Patienten mit zentraler Obstruktion) mit einem hö- heren VDI, da das zu drainierende Volumen höher war bei gleich schneller Drainage wie bei Kontroll-Patienten [8].

Einen zusätzlichen Vorteil bietet die APG für all diese Überlegungen: Zwei objektive Parameter zur Drainage und Wiederauffüllzeit als VDI und VFI werden in einem Untersuchungsdurchlauf erhoben, bei dem der Patient nur einmal sein Bein anheben muss (VDI) und sich dann hinstellen muss (VFI).

\section{Schlussfolgerung}

Die Luft-Plethysmographie (APG) ist ein objektives Untersuchungsinstrument für verzögerte Drainage und inkompetente Venenklappen. Die venöse Insuffizienz wird über den venösen Füllungs-Index (VFI) und die Abflussbehinderung über den venösen Drainage-In$\operatorname{dex}$ (VDI) beurteilt.

Der Test ist nicht invasiv, nicht beeinflusst durch die Fähigkeiten des Patienten oder Untersuchers und ist davon unabhängig, ob eine Behandlung bereits durchgeführt wurde. Er ist einfach durchzuführen und kann in den Alltag der Diagnostik implementiert werden. Dies wäre besonders dann wichtig, wenn wir an einem unabhängigen Follow-up interessiert sind und wenn wir sicher sein wollen, dass tatsächlich eine hämodynamisch relevante Erkrankung vorliegt, bevor wir invasive Therapieansätze wählen. APG sollte vor invasiven Untersuchungsmethoden und auf jeden Fall vor der Einlage eines Stents durchgeführt werden.

\section{Interessenkonflikt}

Die Autoren geben an, dass kein Interessenkonflikt besteht.

\section{Literatur}

[1] Gaweesh AS. Impeded venous drainage: novel view of chronic venous disease pathophysiology. Med Hypotheses 2009; 73: 548-552.

[2] Lattimer CR, Kalodiki E, Mendoza E. Gravitational venous drainage is significantly faster in patients with varicose veins. Phlebology 2016; 31: $546-553$

[3] Kibbe MR, Ujiki M, Goodwin AL, et al. lliac vein com- pression in an asymptomatic patient population. J Vasc Surg 2004; 39: 937-943

[4] Lattimer CR, Mendoza E, Kalodiki E. The current status of air-plethysmography in evaluating non-thrombotic iliac vein lesions. Phlebology 2018; 33: 3-4

[5] Lattimer CR, Kalodiki E, Kafeza M, et al. Quantifying the degree graduated elastic compression stockings enhance venous emptying. Eur J Vasc Endovasc Surg 2014; 47: 75-80

[6] Gianesini S, Zamboni P, Mendoza E. Venous Reflux Patterns in: Zamboni P, Mendoza E, Gianesini S (Eds.); Saphenous Vein-Sparing Strategies in Chronic Venous Disease, Springer, 2018: 35-74

[7] Allan JC. Volume changes in the lower limb in response to postural alterations and muscular exercise. S Afr J Surg 1964; 2: 75-90

[8] Lattimer CR, Mendoza E. Reappraisal of the utility of the tilt-table in the investigation of venous disease. Eur J Vasc Endovasc Surg 2016; 52: 854-861

[9] Pannier F, Gerlach H, Stücker M et al. Leitlinie: Venöse Diagnostik mit der Licht-Reflexions-Rheographie/Photoplethysmographie der Deutschen Gesellschaft für Phlebologie, Phlebologie 2012; 41: 261-263 
[10] Lattimer CR, Doucet S, Geroulakos G et al. Validation of the novel venous drainage index with step-wise increases in thigh compression pressure in the quantification of venous obstruction. J Vasc Surg Venous Lymphat Disord 2017; 5: 88-95

[11] Lattimer CR, Kalodiki E, Azzam M et al. Gravitational venous drainage improves significantly after iliac venous stenting but this may result in faster venous filling. J Vasc Surg Venous Lymphat Disord 2016; 4:137-138

[12] Bradbury A, Evans C, Allan P et al. What are the symptoms of varicose veins? Edinburgh vein study cross sectional population survey. $\mathrm{Br} \mathrm{Med}$ J 1999; 318: 353-356

[13] Blaettler W, Thomae HJ, Amsler F. Venous leg symptoms in healthy subjects assessed during prolonged standing. J Vasc Surg Venous Lymphat Disord 2016; 4: 455-462

[14] Obermayer A, Garzon K. Identifying the source of superficial reflux in venous leg ulcers using duplex ultrasound. J Vasc Surg 2010; 52: 1255-1261

[15] Hareendran A, Bradbury A, Budd J et al. Measuring the impact of venous leg ulcers on quality of life. J Wound Care 2005; 14: 53-57
[16] Villalta S, Bagatella P, Piccioli A et al. Assessment of validity and reproducibility of a clinical scale for the post-thrombotic syndrome (abstract). Haemostasis 1994; 24: 158a

[17] Trinh F, Paolini D, Fish J et al. Use of Villalta score for defining postthrombotic disease may lead to false-positice diagnosis in $42 \%$ of patients with primary chronic venous disease. J Vasc Surg Venous Lymphat Disord 2018; 6: 291

[18] Lattiner CR, Kalodiki E, Azzam M et al. Validation of the Villalta scale in assessing post-thrombotic syndrome using clinical, duplex, and hemodynamic comparators; JVS Venous and Lymphatic Disorders 2014; 2 (1): 8-14

[19] Kurstjens RL, Catarinella FS, Lam YL et al. The inability of venous occlusion air plethysmography to identify patients who will benefit from stenting of deep venous obstruction. Phlebology 2018; 33: 483-491

[20] Gagne PJ, Gasparis A, Black S et al. Analysis of threshold stenosis by multiplanar venogram and intravascular ultrasound examination for predicting clinical improvement after iliofemoral vein stenting in the VIDIO trial. J Vasc Surg Venous Lymphat Disord 2018; 6: 48-56 e1

[21] Vedantham S, Goldhaber SZ, Julian JA et al. Pharmacomechanical catheter-directed thrombolysis for deep-vein thrombosis. N Engl J Med 2017; 377: 2240-2252 